\title{
A Modular Approach to Main-Chain Organometallic Polymers
}

Andrew J. Boydston, Kyle A. Williams, and Christopher W. Bielawski *

Department of Chemistry and Biochemistry, The University of Texas at Austin, Austin, TX 78712

\section{Experimental Section}

Materials and Methods: ${ }^{1} \mathrm{H}$ and ${ }^{13} \mathrm{C}$ NMR spectra were recorded using a Varian Unity Plus 300 or 400 spectrometer. Chemical shifts $(\delta)$ are expressed in ppm downfield from tetramethylsilane using the residual protonated solvent as an internal standard (DMSO- $d_{6},{ }^{1} \mathrm{H} 2.49 \mathrm{ppm}$ and ${ }^{13} \mathrm{C}$ $39.5 \mathrm{ppm}$ ). Coupling constants are expressed in hertz. HRMS (ESI, CI) were obtained with a VG analytical ZAB2-E instrument. UV-vis spectra were recorded using a Perkin Elmer Instruments Lambda 35 spectrometer. GPC data was recorded using an Agilent 1100 HPLC with an Agilent PLgel $5 \mu \mathrm{m}$ MIXED-C column. Molecular weight data is reported relative to polystyrene standards in DMF. $\mathrm{T}_{\mathrm{d}} \mathrm{S}$ were determined using a TA Instruments TGA-Q500 under nitrogen atmosphere. $\mathrm{PhCH}_{3}$ was distilled from $\mathrm{CaH}_{2}$ or sodium and benzophenone under $\mathrm{N}_{2}$ atmosphere prior to use. DMF was used from a solvent purification column under an atmosphere of argon. DMSO for polymerization reactions was of reagent quality and used as obtained from commercial sources.

General Procedure for formation of bis(imidazole)s via condensation of tetraamino arenes with formic acid: A round bottom flask was charged with a magnetic stir bar and the tetraamine (either as free base or tetrahydrochloride salt). Formic acid (88-99\%) was added and the flask was fitted with an air-jacketed condenser. The reaction mixture was placed in an oil bath at 100 ${ }^{\circ} \mathrm{C}$ for 2-36 h. The reaction mixture was then allowed to cool, poured into ice-cold water (equal volume to formic acid) and neutralized with $10 \% \mathrm{NaOH}$ solution or saturated $\mathrm{K}_{2} \mathrm{CO}_{3}$ solution. Neutralization caused precipitation of the product which was collected via vacuum filtration, rinsed with cold water, and dried under vacuum over $\mathrm{P}_{2} \mathrm{O}_{5}$.

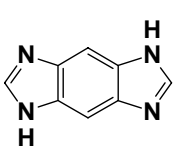

Benzo-bis(imidazole): 1,2,4,5-Benzenetetraamine tetrahydrochloride $1(284 \mathrm{mg}$, $1.00 \mathrm{mmol})$ was treated with formic acid $(10 \mathrm{~mL})$ for $36 \mathrm{~h}$ according to the General Procedure to provide $152 \mathrm{mg}(96 \%)$ of the desired product as a brown solid: $\mathrm{mp}>$ $300 \quad{ }^{\circ} \mathrm{C} ;{ }^{1} \mathrm{H}$ NMR $\left(400 \mathrm{MHz}\right.$, DMSO- $\left.d_{6}\right) \delta 8.16(\mathrm{~s}, 2 \mathrm{H}), 7.67(\mathrm{~s}, 2 \mathrm{H}) ;{ }^{13} \mathrm{C}$ NMR $\left(100 \mathrm{MHz}, \mathrm{DMSO}-\mathrm{d}_{6}\right) \delta 142.5,135.6,99.2 ; \mathrm{HRMS} \mathrm{m} / \mathrm{z}$ calcd for $\mathrm{C}_{8} \mathrm{H}_{7} \mathrm{~N}_{4}\left[\mathrm{M}+\mathrm{H}^{+}\right]$159.0671, found 159.0665 .

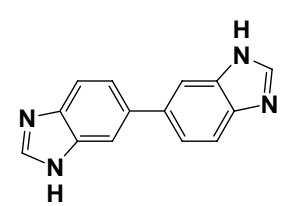

3,3'-bibenzimidazole: 3,3'-diaminobenzidine 3 (4.71 g, $21.98 \mathrm{mmol}$ ) was treated with formic acid $(25 \mathrm{~mL})$ for $2 \mathrm{~h}$ according to the General Procedure to provide $4.92 \mathrm{~g}(96 \%)$ of the desired product as a tan powder. mp $262-267{ }^{\circ} \mathrm{C}$ (dec); ${ }^{1} \mathrm{H}$ NMR (400 MHz, DMSO-d $\left.d_{6}\right) \delta 8.23(\mathrm{~s}, 2 \mathrm{H}), 7.82(\mathrm{~d}, J=1.1 \mathrm{~Hz}, 2 \mathrm{H})$, $7.65(\mathrm{~d}, J=8.3 \mathrm{~Hz}, 2 \mathrm{H}), 7.51(\mathrm{dd}, J=8.3,1.1 \mathrm{~Hz}, 2 \mathrm{H}) ;{ }^{13} \mathrm{C} \mathrm{NMR}(100 \mathrm{MHz}$, 
DMSO- $\left.d_{6}\right) \delta 143.0,138.7,137.9,135.9,122.0,116.1,113.5 ; \mathrm{HRMS} \mathrm{m} / \mathrm{z}$ calcd for $\mathrm{C}_{14} \mathrm{H}_{11} \mathrm{~N}_{4}$ $\left[\mathrm{M}+\mathrm{H}^{+}\right]$235.0984, found 235.0985.

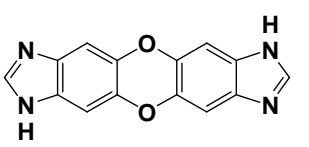

Dioxin-bis(imidazole): Dioxin-2,3,7,8-tetraamine tetrahydrochloride (5) $(525 \mathrm{mg}, 1.35 \mathrm{mmol})$ was treated with formic acid $(15 \mathrm{~mL})$ for $3 \mathrm{~h}$ according to the General Procedure (complete dissolution was not achieved, and the reaction mixture remained a suspension) to provide $303 \mathrm{mg}(85 \%)$ of the desired product as a tan powder: $\mathrm{mp}>300{ }^{\circ} \mathrm{C}(\mathrm{dec}) ;{ }^{1} \mathrm{H}$ NMR $\left(400 \mathrm{MHz}, \mathrm{DMSO}-d_{6}\right) \delta 8.13(\mathrm{~s}, 2 \mathrm{H}), 8.11(\mathrm{~s}, 2 \mathrm{H}), 7.21(\mathrm{~s}$, $4 \mathrm{H}) ;{ }^{13} \mathrm{C}$ NMR $\left(100 \mathrm{MHz}, \mathrm{DMSO}-d_{6}\right) \delta 163.2,142.7,138.4,101.8 ; \mathrm{HRMS} \mathrm{m} / \mathrm{z}$ calcd for $\mathrm{C}_{14} \mathrm{H}_{9} \mathrm{~N}_{4} \mathrm{O}_{2}\left[\mathrm{M}+\mathrm{H}^{+}\right]$265.0726, found 265.0725.

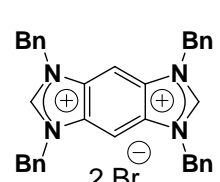

Tetrabenzyl benzo-bis(imidazolium) bromide (2a): To a solution of $\mathrm{NaH}$ (298 $\mathrm{mg}, 60 \mathrm{wt} \%, 7.46 \mathrm{mmol})$ in $\mathrm{PhCH}_{3}(30 \mathrm{~mL})$ was added the corresponding bis(imidazole) (576 mg, $3.64 \mathrm{mmol})$ under dry nitrogen. The solution was heated $\mathrm{Bn} 2 \mathrm{Br} \quad \mathrm{Bn}$ to $110^{\circ} \mathrm{C}$ for $1 \mathrm{~h}$, allowed to cool to ambient, and benzylbromide $(2.61 \mathrm{~mL}, 21.84$ mmol) was added via syringe. The suspension was placed in an oil bath at $110^{\circ} \mathrm{C}$ for $1 \mathrm{~h}$, then dry DMF $(30 \mathrm{~mL})$ was added via syringe and the reaction was maintained at $110{ }^{\circ} \mathrm{C}$ for $6 \mathrm{~h}$, then $60{ }^{\circ} \mathrm{C}$ for $4 \mathrm{~h}$. Upon completion, the suspension was allowed to cool, diluted with $\mathrm{PhCH}_{3}(50 \mathrm{~mL})$ and the solids were collected by vacuum filtration, rinsed with water and THF successively, and dried under vacuum over $\mathrm{P}_{2} \mathrm{O}_{5}$ to give $2.42 \mathrm{~g}(98 \%)$ as a tan powder: mp $280-282{ }^{\circ} \mathrm{C}(\mathrm{dec}) ;{ }^{1} \mathrm{H}$ NMR (400 MHz, DMSO-d $)) \delta 10.54(\mathrm{~s}, 2 \mathrm{H}), 8.93(\mathrm{~s}, 2 \mathrm{H}), 7.60-7.58(\mathrm{~m}, 8 \mathrm{H}), 7.39-7.34(\mathrm{~m}$, $12 \mathrm{H}), 5.87(\mathrm{~s}, 8 \mathrm{H}),{ }^{13} \mathrm{C}$ NMR $\left(100 \mathrm{MHz}, \mathrm{DMSO}-d_{6}\right) \delta 146.1,133.4,129.9,128.9,128.8,128.6$, 99.8, 50.5; HRMS m/z calcd for $\mathrm{C}_{36} \mathrm{H}_{31} \mathrm{~N}_{4}\left[\mathrm{M}-\mathrm{H}^{+}\right]$519.2549, found 519.2543.

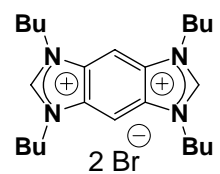

Tetrabutyl benzo-bis(imidazolium) bromide (2b): It should be noted that $\mathbf{2 b}$ is $\mathrm{H}_{2} \mathrm{O}$-soluble. To a solution of $\mathrm{NaH}(415 \mathrm{mg}, 60 \mathrm{wt} \%, 10.37 \mathrm{mmol})$ in $\mathrm{PhCH}_{3}(20$ $\mathrm{mL})$ was added the corresponding bis(imidazole) $(800 \mathrm{mg}, 5.06 \mathrm{mmol})$ under argon and the resulting suspension was placed in an oil bath at $110{ }^{\circ} \mathrm{C}$ for $1 \mathrm{~h}$. To the cooled suspension was added 1-bromobutane $(3.26 \mathrm{~mL}, 30.36 \mathrm{mmol})$ via syringe and the mixture was heated at $110{ }^{\circ} \mathrm{C}$ for $1 \mathrm{~h}$, then dry DMF $(20 \mathrm{~mL})$ was added via syringe and the mixture was stirred at $110^{\circ} \mathrm{C}$ for $8 \mathrm{~h}$, then $60{ }^{\circ} \mathrm{C}$ for $4 \mathrm{~h}$. The cooled reaction mixture was then concentrated under vacuum and the residue taken up in $1: 1 \mathrm{CH}_{2} \mathrm{Cl}_{2} / \mathrm{MeOH}$ and the insoluble $\mathrm{NaBr}$ was removed via filtration. To the filtrate was added EtOAc until precipitation of the product occurred. The solids were collected via vacuum filtration and dried under vacuum to provide $2.66 \mathrm{~g}(97 \%)$ of the product as a tan powder: $\mathrm{mp} 194-198{ }^{\circ} \mathrm{C}(\mathrm{dec}) ;{ }^{1} \mathrm{H} \mathrm{NMR}(300 \mathrm{MHz}$, DMSO-d $)_{6} \delta 10.25(\mathrm{~s}, 2 \mathrm{H}), 9.11(\mathrm{~s}, 2 \mathrm{H}), 4.62(\mathrm{t}, J=7.4 \mathrm{~Hz}, 8 \mathrm{H}), 1.97(\mathrm{p}, J=7.4 \mathrm{~Hz}, 8 \mathrm{H}), 1.38$ (sext, $J=7.4 \mathrm{~Hz}, 8 \mathrm{H}), 0.93(\mathrm{t}, J=7.4 \mathrm{~Hz}, 12 \mathrm{H}) ;{ }^{13} \mathrm{C}$ NMR $\left(75 \mathrm{MHz}, \mathrm{DMSO}-d_{6}\right) \delta 145.5,130.2$, 99.3, 47.1, 30.4, 19.1, 13.5; HRMS m/z calcd for $\mathrm{C}_{24} \mathrm{H}_{39} \mathrm{~N}_{4}\left[\mathrm{M}-\mathrm{H}^{+}\right]$383.3175, found 383.3180.

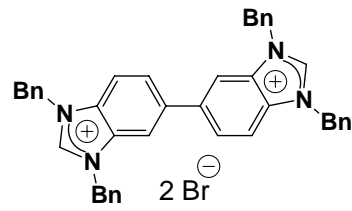

Tetrabenzyl bis(benzimidazolium) bromide (4a): In a manner analogous to 2a, $3.05 \mathrm{~g}(98 \%)$ of compound $4 \mathbf{a}$ was obtained as a tan solid: $\mathrm{mp} 179-183{ }^{\circ} \mathrm{C}(\mathrm{dec}) ;{ }^{1} \mathrm{H}$ NMR $\left(400 \mathrm{MHz}, \mathrm{DMSO}-d_{6}\right) \delta 10.29(\mathrm{~s}, 2 \mathrm{H})$, $8.67(\mathrm{~s}, 2 \mathrm{H}), 8.1(\mathrm{~d}, J=8.8 \mathrm{~Hz}, 2 \mathrm{H}), 8.04(\mathrm{~d}, J=8.8 \mathrm{~Hz}, 2 \mathrm{H}), 7.66(\mathrm{~d}, J=$ $7.2 \mathrm{~Hz}, 4 \mathrm{H}), 7.57$ (d, $J=7.2 \mathrm{~Hz}, 4 \mathrm{H}) 6.00(\mathrm{~s}, 4 \mathrm{H}), 5.87 ;{ }^{13} \mathrm{C}$ NMR $(100$ 
MHz, DMSO-d $\left.d_{6}\right) \delta 143.5,137.7,134.1,134.0,131.9,130.8,129.01,128.97,128.7,128.5,128.3$, 126.5, 114.7, 112.9, 50.1, 50.0; HRMS m/z calcd for $\mathrm{C}_{42} \mathrm{H}_{35} \mathrm{~N}_{4}\left[\mathrm{M}-\mathrm{H}^{+}\right]$595.2862, found 595.2858 .

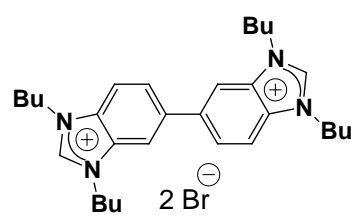

Tetrabutyl bis(benzimidazolium) bromide (4b): It should be noted that 4b is $\mathrm{H}_{2} \mathrm{O}$-soluble. In a manner analogous to $\mathbf{2 b}, 1.96 \mathrm{~g}(74 \%)$ of compound 4b was obtained as a tan solid: $\mathrm{mp} 248-255{ }^{\circ} \mathrm{C}(\mathrm{dec}) ;{ }^{1} \mathrm{H} \mathrm{NMR}$ $\left(400 \mathrm{MHz}, \mathrm{DMSO}-d_{6}\right) \delta 10.05(\mathrm{~s}, 2 \mathrm{H}), 8.71(\mathrm{~s}, 2 \mathrm{H}), 8.28(\mathrm{~d}, J=8.7 \mathrm{~Hz}$, $2 \mathrm{H}), 8.2(\mathrm{dd}, J=8.7,1.4 \mathrm{~Hz}, 2 \mathrm{H}), 4.66(\mathrm{t}, J=7.2 \mathrm{~Hz}, 4 \mathrm{H}), 4.57(\mathrm{t}, J=7.2$ $\mathrm{Hz}, 4 \mathrm{H}), 1.97-1.90(\mathrm{~m}, 8 \mathrm{H}), 1.41-1.32(\mathrm{~m}, 8 \mathrm{H}), 0.93(\mathrm{t}, J=7.2 \mathrm{~Hz}, 12 \mathrm{H}) ;{ }^{13} \mathrm{C} \mathrm{NMR}(100 \mathrm{MHz}$, DMSO- $\left.d_{6}\right) \delta 142.9,137.5,132.0,130.9,126.3,114.3,112.6,46.6,34.2,30.67,30.65,19.09$, 19.07, 13.5, 13.4; HRMS m/z calcd for $\mathrm{C}_{30} \mathrm{H}_{45} \mathrm{~N}_{4}\left[\mathrm{M}+\mathrm{H}^{+}\right]$461.3644, found 461.3643.

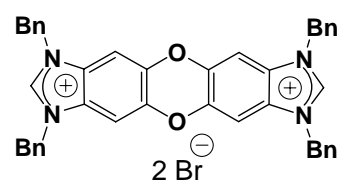

Tetrabenzyl dioxin-bis(imidazolium) bromide (6): In a manner analogous to 2a, $707 \mathrm{mg}(90 \%)$ of the compound $\mathbf{6}$ was obtained as a yellow-tan powder: mp 252-258 ${ }^{\circ} \mathrm{C}(\mathrm{dec}) ;{ }^{1} \mathrm{H} \mathrm{NMR}\left(400 \mathrm{MHz}, \mathrm{DMSO}-d_{6}\right)$ $\delta 10.09(\mathrm{~s}, 2 \mathrm{H}), 7.77(\mathrm{~s}, 4 \mathrm{H}), 7.56-7.55(\mathrm{~m}, 8 \mathrm{H}), 7.44-7.38(\mathrm{~m}, 12 \mathrm{H}), 5.73$ $(\mathrm{s}, 8 \mathrm{H}) ;{ }^{13} \mathrm{C}$ NMR $\left(100 \mathrm{MHz}, \mathrm{DMSO}-d_{6}\right) \delta 142.8,140.7,133.8,129.0,128.8,128.4,127.4,101.2$, 50.1; HRMS m/z calcd for $\mathrm{C}_{42} \mathrm{H}_{33} \mathrm{~N}_{4} \mathrm{O}_{2}\left[\mathrm{M}-\mathrm{H}^{+}\right]$625.2604, found 625.2601.

Typical procedure for polymerization: Polymerizations were conducted on $0.1-2.0 \mathrm{~g}$ scale. Bis(imidazolium) bromide (1.0 eqv) was dissolved in DMSO (ca. 0.1-0.2 M) and either $\mathrm{M}^{(\mathrm{II})}(\mathrm{OAc})_{2}$ (1.0 eqv), or a combination of $\mathrm{M}^{(\mathrm{II})} \mathrm{Cl}_{2}$ (1.0 eqv) and $\mathrm{NaOAc}$ (2.0 eqv), was added in one portion. The solution was placed in a preheated oil bath at $110{ }^{\circ} \mathrm{C}$ and stirred open-air for 1 $5 \mathrm{~h}$. The reaction mixtures typically darkened in color (to orange-brown) as the reaction progressed. In the case of $\mathbf{8 a}$ and $\mathbf{9 a}$ a green reaction mixture was eventually obtained. The cooled reaction mixture was precipitated into either $\mathrm{MeOH}$ or $\mathrm{H}_{2} \mathrm{O}$ and the solids were collected via vacuum filtration and dried under vacuum.

7a: Light brown solid. ${ }^{1} \mathrm{H}$ NMR (400 MHz, DMSO-d $) \delta$ 7.80-6.80 (m, 22H), 6.40-5.41 (m, 8H); $\mathrm{T}_{\mathrm{d}}=291{ }^{\circ} \mathrm{C} ; \mathrm{M}_{n}=18.4 \times 10^{3} ; \mathrm{PDI}=1.9$.

7b: Tan solid. ${ }^{1} \mathrm{H}$ NMR (400 MHz, DMSO-d $\left.{ }_{6}\right) \delta 8.20-8.04(\mathrm{~m}, 2 \mathrm{H}), 4.95-4.76(\mathrm{~m}, 8 \mathrm{H}), 2.18-$ $2.10(\mathrm{~m}, 8 \mathrm{H}), 1.48-1.40(\mathrm{~m}, 8 \mathrm{H}), 1.01-0.92(\mathrm{~m}, 12 \mathrm{H}) ; \mathrm{M}_{n}=103 \times 10^{3} ; \mathrm{PDI}=1.8$.

7c: Tan solid. ${ }^{1} \mathrm{H}$ NMR (400 MHz, DMSO-d $\left.{ }_{6}\right) \delta$ 7.60-6.45 (m, 26H), 6.40-5.20 (m, 8H); $\mathrm{T}_{\mathrm{d}}=$ $294{ }^{\circ} \mathrm{C} ; \mathrm{M}_{n}=1760 \times 10^{3} ; \mathrm{PDI}=1.3$.

7d: Tan powder. ${ }^{1} \mathrm{H}$ NMR (400 MHz, DMSO- $\left.d_{6}\right) \delta 8.18$ (br s, 2H), 4.91-4.55 (m, 8H), 2.18-1.99 $(\mathrm{m}, 8 \mathrm{H}), 1.47$ (br s, 8H), $1.00-0.91(\mathrm{~m}, 12 \mathrm{H}) ; \mathrm{T}_{\mathrm{d}}=295^{\circ} \mathrm{C} ; \mathrm{M}_{n}=766 \times 10^{3} ; \mathrm{PDI}=1.7$.

8a: Light green powder. ${ }^{1} \mathrm{H}$ NMR (400 MHz, DMSO-d $) \delta 7.62$ (br s, 7H), 7.40-6.90 (m, 19H), 6.13 (br s, $4 \mathrm{H}), 6.07$ (br s, $4 \mathrm{H}) ; \mathrm{T}_{\mathrm{d}}=284^{\circ} \mathrm{C} ; \mathrm{M}_{n}=26.1 \times 10^{3}$; PDI $=2.0$.

8b: Yellow solid. ${ }^{1} \mathrm{H}$ NMR (400 MHz, DMSO- $\left.d_{6}\right) \delta$ 7.97-7.92 (m, 2H), 7.77-7.60 (m, 4H), 4.83$4.54(\mathrm{~m}, 8 \mathrm{H}), 2.18-2.14(\mathrm{~m}, 8 \mathrm{H}), 1.50($ br s, $8 \mathrm{H}), 0.99($ br s, $12 \mathrm{H}) ; \mathrm{M}_{n}=8.00 \times 10^{3}$; PDI $=1.4$. 
9a: Green powder. ${ }^{1} \mathrm{H}$ NMR $\left(400 \mathrm{MHz}, \mathrm{DMSO}-\mathrm{d}_{6}\right) \delta$ 7.80-6.90 $(\mathrm{m}, 26 \mathrm{H}), 6.20-5.90(\mathrm{~m}, 8 \mathrm{H}) ; \mathrm{T}_{\mathrm{d}}$ $=299^{\circ} \mathrm{C} ; \mathrm{M}_{n}=106 \times 10^{3} ; \mathrm{PDI}=1.5$.

9c: Tan powder. ${ }^{1} \mathrm{H}$ NMR $\left(400 \mathrm{MHz}, \mathrm{DMSO}_{6}\right) \delta$ 7.80-7.00 $(\mathrm{m}, 26 \mathrm{H}), 6.40-5.40(\mathrm{~m}, 8 \mathrm{H}) ; \mathrm{M}_{n}=$ $408 \times 10^{3}$; PDI $=2.6$. 